\title{
Design optimisation and resource assessment for tidal-stream renewable energy farms using a new continuous turbine approach
}

\author{
S.W. Funke ${ }^{\mathrm{a},}$, S.C. Kramer ${ }^{\mathrm{b}}$, M.D. Piggott ${ }^{\mathrm{b}, \mathrm{c}}$ \\ ${ }^{a}$ Center for Biomedical Computing, Simula Research Laboratory, Oslo, Norway \\ ${ }^{b}$ Applied Modelling and Computation Group, Department of Earth Science and \\ Engineering, Imperial College London, London, UK \\ ${ }^{c}$ Grantham Institute for Climate Change, Imperial College London, London, UK
}

\begin{abstract}
This paper presents a new approach for optimising the design of tidal stream turbine farms. In this approach, the turbine farm is represented by a turbine density function that specifies the number of turbines per unit area and an associated continuous locally-enhanced bottom friction field. The farm design question is formulated as a mathematical optimisation problem constrained by the shallow water equations and solved with efficient, gradient-based optimisation methods. The resulting method is accurate, computationally efficient, allows complex installation constraints, and supports different goal quantities such as to maximise power or profit. The outputs of the optimisation are the optimal number of turbines, their location within the farm, the overall farm profit, the farm's power extraction, and the installation cost.

We demonstrate the capabilities of the method on a validated numerical model of the Pentland Firth, Scotland. We optimise the design of four tidal farms simultaneously, as well as individually, and study how farms in close proximity may impact upon one another.
\end{abstract}

Keywords: marine renewable energy, tidal stream turbines, optimisation, shallow water equations, resource assessment, Pentland Firth

\section{Introduction}

Tidal stream turbines represent one of the most promising technologies for capturing marine energy. These turbines are installed in fast tidally-induced currents, where they convert mechanical energy into electricity in a similar way to wind turbines. Farms comprising hundreds of turbines have the potential to produce hundreds of megawatts of highly predictable power. However, in order to be economically viable, these farms must be carefully designed. Two of the

Email address: simon@simula.no (S.W. Funke) 
most critical design decisions are (i) the locations of the turbines, and (ii) the number of turbines making up the farm.

Finding good turbine locations is challenging for a number of reasons. Firstly, various practical constraints need to be taken into account that pose limitations on where turbines may be installed, for instance turbines may not be installed in areas which are too steep, shallow or deep. Secondly, a tidal turbine has both local effects on the flow (for instance reduced flow speed downstream of the turbine due to wake effects, and accelerated flow to the sides of the swept area), and basin-wide effects (reduced flow speeds due to large-scale blockage effects). Furthermore, the turbine's energy output depends cubically on the flow speed. Combining these effects means that the performance of each turbine depends on the position of all other turbines, and that even small changes in a turbine's location may result in a significant change in the total farm performance (Sutherland et al., 2007; Polagye and Malte, 2011; Draper et al., 2013; Funke et al., 2014). At larger geographical scales, the design of an individual array may have implications for the optimal location, size and design of other arrays in the vicinity. It is therefore potentially important to consider both inter- and intra- array design and competition issues in a fully coupled manner.

The optimal number of turbines in a farm is the result of multiple competing factors. Adding turbines generally (up to a point) increases the farm's total power production, but also increases the total project cost. Furthermore, the energy available to a farm should be considered as a limited resource, which means that the power production per turbine decreases with increasing numbers of turbines (Vennell et al. 2015). As a result, the overall profitability of the farm reduces if too few or too many turbines are installed in the farm.

When combining all these factors it becomes clear that determining the best farm design is a complex, tightly coupled optimisation problem. The simplest approach to tackle this problem is to select some potential designs and to use a hydrodynamic model to predict their performance (Lee et al. 2010; Ahmadian and Falconer, 2012; Divett et al., 2013). However, in practice the number of possible designs is too large to be fully explored. Another option is to simplify the hydrodynamic model such that an analytical solution can be derived. Then all possible farm designs can be analysed and the optimum design derived directly (Garrett and Cummins, 2005, Vennell, 2010, 2011). A more recent approach uses an accurate hydrodynamic model and applies efficient, derivativebased optimisation methods to determine optimal farm designs (Funke et al. 2014, Culley et al., 2016, Barnett et al., 2015).

This paper presents a new continuous turbine approach for designing tidal turbine farms and for resource assessment. The basic idea is similar to Funke et al. (2014) in that we formulate the farm design problem as a mathematical optimisation problem, describe the hydrodynamics by the shallow water equations, and solve the problem with derivative-based optimisation methods. The novelty of this work is that the tidal farm is represented as a turbine density function, in contrast to resolving individual turbines as in Funke et al. (2014). This turbine density function has high values in areas with many tidal turbines and vanishes in areas with no turbines. 
The proposed approach offers a number of key benefits. It is accurate, because the interaction between farm and flow is treated in a fully coupled manner, here through the solution of the shallow water equations. It is flexible in that it naturally supports minimum distance constraints between turbines, feasible turbine locations can be chosen arbitrarily, and the goal quantity defining the optimisation problem can be a combination of farm profit, installation cost, and other user-defined quantities. Furthermore, the approach is efficient because the total number as well as the positions (or rather local density) of the turbines are simultaneously optimised for, because the numerical mesh does not need to resolve individual turbines, and because the derivative-based optimisation methods scale well with the farm size. In fact, from our experience computational cost is nearly independent on the farm size and the number of turbines. As a result, this approach is suitable for designing large farms with hundreds of turbines and complex constraints, and further allows for the design of multiple (potentially interacting and competing) farms simultaneously.

More specifically, the novel contributions of this work are the following:

- We formulate the turbine farm design problem and resource assessment in terms of a mathematical optimisation problem. The farm is represented by a turbine density function (sections 244 . The goal is to identify the farm configuration which maximises the farm's financial profit.

- We show how to discretise, and efficiently solve this optimisation problem using derivative-based optimisation algorithms and the adjoint equations (section 5). The implementation is available as part of the opensource software OpenTidalFarm (opentidalfarm.org).

- We show that the results from the new continuous approach are consistent with those obtained using the approach presented in Funke et al. (2014), where turbines were resolved explicitly (section 6).

- We validate the underlying hydrodynamic model against observations made in the Pentland Firth (section 7).

- We demonstrate the efficiency of the approach by optimising four turbine farms in the Pentland Firth, first individually and then simultaneously, and compare the farm designs (section 8). We show that farms have the potential to significantly impact one another, and that this interaction should be taken into account during the design process. To our knowledge this is the first example of a simultaneous multi-farm design optimisation.

\section{Problem formulation}

\subsection{Farm optimisation}

We formulate the farm design (the total number and positions of turbines) question via the optimisation problem: 

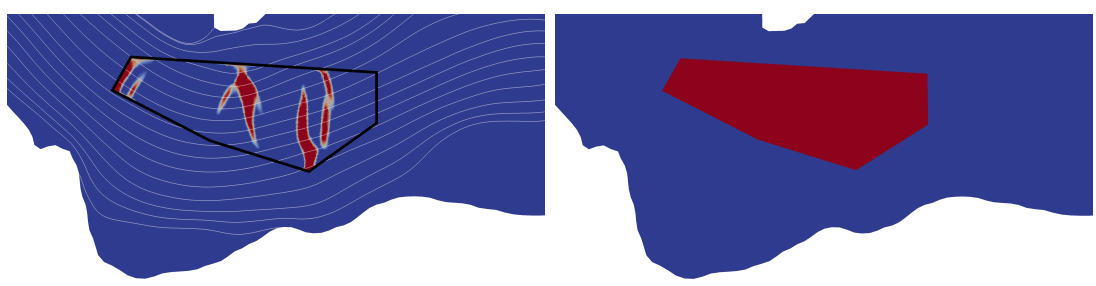

Figure 1: Solution of the tidal farm design problem (1) for a site in the Inner Sound of the Pentland Firth. The image on the left shows the optimal turbine density field $d(x)$ with flow streamlines and farm boundary indicated. The image on the right shows the upper density bound $\bar{d}$, which here takes the value zero outside the array area. The optimisation predicts an optimal number of 266 turbines, and a farm power production of $316 \mathrm{MW}$. Only a subset of the entire computational domain is shown here, refer to section 8 for further details.

$$
\begin{aligned}
& \max _{d} \operatorname{Profit}(d) \\
& \text { subject to } 0 \leq d \leq \bar{d} .
\end{aligned}
$$

Here, 'Profit (.)' is a function which evaluates the financial profit of the farm over its entire lifetime and is the goal quantity to be maximised. The farm design is described by a spatially varying turbine density function $d: \Omega \rightarrow \mathbb{R}$ (e.g. figure 1. left), where $\Omega \subset \mathbb{R}^{2}$ is the horizontal domain. That is, an area with $d(\vec{x})=0$ has no turbines, and an area with $d(\vec{x})=\bar{d}(\vec{x})$ has densely deployed turbines. The upper bound $\bar{d}$ is defined by the user and specifies the maximum number of turbines per unit area: it is zero where no turbines may be installed (outside the lease area, or in too steep, shallow or deep areas as shall be demonstrated in section 8), and a positive constant elsewhere (e.g. figure 1, right). This constant could be derived from a dense grid layout: for a minimal distance $D_{\min }$ between turbines it is $\bar{d}(\vec{x})=1 / D_{\min }^{2}$. This could trivially be extended to account for different inter- and intra- row spacing constraints.

The solution of the farm design problem (1) yields several useful insights. Firstly, the optimal turbine density field $d(x)$ indicates where the turbines should be installed. Secondly, the optimal number of turbines $N$ is obtained by integrating the optimal density function, that is

$$
N=\int_{\Omega} d(\vec{x}) \mathrm{d} \vec{x}
$$

Finally, the Profit function provides a good indicator for the potential of the tidal resource for a given farm site.

\subsection{Profit model}

A profit model for a tidal farm can be very complex and include various factors such as the farm design, turbine properties, installation costs, learning 
rates and the underlying energy market (Roc et al. 2015). Here, we consider for simplicity only two dominant components: the revenue of selling the produced energy and the cost of buying, installing and maintaining the turbines, that is:

$$
\operatorname{Profit}(d)=\operatorname{Revenue}(d)-\operatorname{Cost}(d) .
$$

The revenue of the tidal farm over its lifetime is modelled here as

$$
\operatorname{Revenue}(d)=\operatorname{IkE}(\vec{u}, d),
$$

where $I$ denotes the financial income per energy unit, $0 \leq k \leq 1$ is a turbine efficiency coefficient that takes mechanical and electrical losses into account, and $E(\vec{u}, d)$ is the total amount of energy that the farm (encoded by $d$ ) extracts from the flow velocity $\vec{u}$ over its lifetime. Note that $\vec{u}$ can itself be considered a function of $d$.

A very simple cost model is employed here that scales linearly with the number of installed turbines:

$$
\operatorname{Cost}(d)=C \int_{\Omega} d(\vec{x}) \mathrm{d} \vec{x},
$$

where $C$ is the financial cost of a single turbine. Given (2) this of course is equivalent to Cost $=C N$. In the special case of setting the turbine cost to zero, the solution to (1) yields the maximum extractable energy from the farm site.

Note that the Revenue and Cost models do not take a discount rate into account, and no distinction is made between upfront investments and e.g. maintenance costs in the Cost model. Although these are essential factors to obtain a realistic estimate of the profitability of a farm, these have been left out here for simplicity but could easily be incorporated when realistic values are known.

\section{The energetics of a tidal turbine farm}

\subsection{Shallow water equations}

The farm energy extraction $E(\vec{u}, d)$ depends on the flow velocity $\vec{u}$, which in turn depends on the farm design $d$. Here we model these dynamics using the time-dependent, nonlinear shallow water equations with the presence of turbines represented through a locally-increased bottom friction coefficient. The resulting equations are

$$
\begin{gathered}
\frac{\partial \vec{u}}{\partial t}+\vec{u} \cdot \nabla \vec{u}-\nu \nabla^{2} \vec{u}+g \nabla \eta+\frac{c_{b}+c_{t}(d)}{H}\|\vec{u}\| \vec{u}=0, \\
\frac{\partial \eta}{\partial t}+\nabla \cdot(H \vec{u})=0,
\end{gathered}
$$

with appropriate initial and boundary conditions. Here $\vec{u}: \Omega \times(0, T) \rightarrow \mathbb{R}^{2}$ is the depth-averaged velocity, $\eta: \Omega \times(0, T) \rightarrow \mathbb{R}$ is the free-surface displacement, $H: \Omega \rightarrow \mathbb{R}$ is the total water depth (that is $H=\eta+h$, where $h$ is the water depth 
at rest), $c_{b}: \Omega \rightarrow \mathbb{R}$ is the natural background friction which here is assumed to be constant, and $c_{t}(d): \Omega \rightarrow \mathbb{R}$ is the additional farm induced friction field that represents the drag exerted by the turbine farm on the flow. The viscosity term, with kinematic viscosity $\nu: \Omega \rightarrow \mathbb{R}$, represents mixing through sub-grid scale processes. A fixed eddy viscosity has been chosen in the runs presented here.

\subsection{Relationship between turbine density and friction}

To derive the relationship between the farm induced friction $c_{t}$ and the turbine density function $d$, we use an idea very similar to the enhanced bottom drag formulations (Divett et al., 2013, Funke et al., 2014; Martin-Short et al. 2015), where the turbine induced drag is chosen such that the resulting force approximates the drag force of an analytical model of the turbines.

To calculate the force associated with the farm induced friction $c_{t}$, we consider the depth-integrated momentum equation which can be derived from the shallow water equations (5):

$$
\rho\left(\frac{\partial H \vec{u}}{\partial t}+\nabla \cdot(H \vec{u} \otimes \vec{u})-\nu H \nabla^{2} \vec{u}+g H \nabla \eta\right)+\rho\left(c_{b}+c_{t}(d)\right)\|\vec{u}\| \vec{u}=0 .
$$

When integrated over some arbitrary area, the first term represents the change of momentum in that area. The integration of the subsequent terms gives sources and sinks in this momentum balance, for instance the integrated advection term can be written in terms of momentum fluxes into and out of the area. The integral of the friction term gives a momentum sink. Thus, the force produced by the farm induced friction $c_{t}$ is given by:

$$
\mathbf{F}_{\text {farm }}(t)=\rho \int_{\Omega} c_{t}(d(\vec{x}))\|\vec{u}(\vec{x}, t)\| \vec{u}(\vec{x}, t) \mathrm{d} \vec{x} .
$$

We now consider a single turbine in a three-dimensional flow with upstream velocity $\vec{u}_{\infty}$. Its drag force $\mathbf{F}_{\text {turbine }} \in \mathbb{R}^{3}$ is commonly parameterised as

$$
\mathbf{F}_{\text {turbine }}\left(\vec{u}_{\infty}\right)=\frac{1}{2} \rho C_{t} A_{t}\left\|\vec{u}_{\infty}\right\| \vec{u}_{\infty}
$$

where $C_{t}$ is the (dimensionless) drag coefficient of the turbine, $A_{t}$ is the surface area swept by the turbine's blades, and $\vec{u}_{\infty}$ is the free-stream velocity. The drag of a farm made up of $N$ devices is therefore given by:

$$
\mathbf{F}_{\text {farm }}=\sum_{i=1}^{N} \frac{1}{2} \rho C_{t} A_{t}\left\|\vec{u}_{\infty, i}\right\| \vec{u}_{\infty, i} .
$$

Here we assume that every turbine has the same thrust coefficient $C_{t}$ and crosssectional surface area $A_{t}$ but take into account a different free-stream velocity $\vec{u}_{\infty, i}$. The interpretation of free-stream velocity in the case of multiple interdependent turbines is not straight-forward. For resolved three-dimensional models this issue can be circumvented by rewriting the force as a function of the 
turbine velocity (Roc et al., 2015). In depth-averaged models however, the local velocity available in the model will be somewhere between the free-stream and turbine velocities, based both on vertical considerations as well as the fact that the less well-resolved the model is in the horizontal (and therefore the more the friction is 'spread out' over the computational mesh) the closer the local model velocity is to the idealised free-stream velocity (see Kramer and Piggott (2015) for a more detailed discussion). In the turbine density approach proposed here, individual turbines are not in any sense individually resolved and therefore the local model velocity at the turbine location may be a good approximation to the free-stream velocity.

Next, we turn the farm drag force from a sum over the discrete turbines into an integral of continuous drag scaled by the turbine density function $d$ :

$$
\mathbf{F}_{\text {farm }}(t)=\int_{\Omega} \frac{1}{2} \rho C_{T} A_{t} d(\vec{x})\|\vec{u}(\vec{x}, t)\| \vec{u}(\vec{x}, t) \mathrm{d} \vec{x} .
$$

Comparing (6) with (7), we see that to obtain the desired farm drag, we need to set

$$
c_{t}(d(\vec{x}))=\frac{1}{2} C_{T} A_{T} d(\vec{x}) .
$$

\subsection{Farm production}

The farm's energy extraction, $E$, in (4) is computed from the hydrodynamic model. Taking the dot product of $(6)$ with $\vec{u}$ yields the farm's power extraction from the flow:

$$
P_{\text {farm }}(t)=\rho \int_{\Omega} c_{t}(d(\vec{x}))\|\vec{u}(\vec{x}, t)\|^{3} \mathrm{~d} \vec{x} .
$$

The total energy extraction is obtained by integrating the power over the farm's lifetime $L$ :

$$
E(\vec{u}, d)=\rho \int_{0}^{L} \int_{\Omega} c_{t}(d(\vec{x}))\|\vec{u}(\vec{x}, t)\|^{3} \mathrm{~d} \vec{x} \mathrm{~d} t .
$$

It should be noted that the energy extracted from the flow by the enhanced bottom drag term will generally be an over-estimate of the energy that can be directly taken out of the flow by the turbines; part of the energy loss is due to mixing losses (e.g. vertical mixing) that are not explicitly represented in the model (Kramer and Piggott, 2015). To compensate for this in the Revenue model, the percentage of usefully extractable energy can be incorporated in the factor $k$ in (4).

\subsection{Blockage effects and rating}

For a free standing turbine the theoretical maximum for the thrust coefficient is given by the Lanchester-Betz limit: $C_{T}=8 / 9$. Depending on the ratio of the turbine cross-section with respect to the cross-section of the farm over its entire width (local blockage) and with respect to the total channel cross section (global blockage) however, this limit can be considerably higher. These blockage effects are not correctly incorporated in the depth-averaged approach 
with constant $C_{T}$ described here because the drag is effectively applied over the entire water depth, and in particular local blockage effects cannot be explicitly modelled. Several papers have derived analytical expressions (see e.g. Garrett and Cummins (2008); Whelan et al. (2009); Nishino and Willden (2012)) that calculate blockage effects in idealized circumstances. It is a matter of ongoing research to investigate whether these expressions can be incorporated within the depth-averaged drag approach to correct for blockage effects. See Vogel (2014) for an example of a blockage correction applied to a tidal fence in a depth-averaged model.

Another effect that is not taken into account by using a constant $C_{T}$ is the fact that turbines are typically tuned to maintain a constant power output above a certain rated speed, by dropping the effective thrust coefficient with increasing speed. Thus instead of a constant $C_{T}$, a thrust curve should be considered, i.e. $C_{T}(\vec{u})$ is a function of the flow speed $\vec{u}$, and the thrust force is no longer quadratic. Exact details for turbines that are expected to be installed in the sites that are investigated in this paper are hard to obtain. For simplicity we therefore follow the constant $C_{T}$ approach described above. It should be noted however that when these details are known, the optimisation approach described in this paper can be easily extended to use generic thrust curves. Likewise if algebraic expressions can be derived to incorporate blockage effects more accurately - this is a matter of further research - such expressions can also easily be incorporated in the optimisation procedure.

\section{Formulation as an optimisation problem}

Reconsider the profit model (3):

$$
\operatorname{Profit}(d)=\operatorname{IkE}(\vec{u}, d)-C \int_{\Omega} d(\vec{x}) \mathrm{d} \vec{x} .
$$

Unfortunately, determining the cost of a turbine $C$, the income factor $I$ and the turbine efficiency coefficient $k$ requires detailed financial and technical specification of tidal turbines and predictions of the electricity market, which are difficult to acquire or estimate. However, the necessary information can be condensed into a single coefficient by observing that the solution of problem (1) is invariant with respect to scaling. That is we can divide the goal quantity (10) by LIk and obtain the full continuous farm optimisation problem:

$$
\max _{d} \frac{1}{L} E(\vec{u}, d)-\frac{C}{L I k} \int_{\Omega} d(\vec{x}) \mathrm{d} \vec{x}
$$

subject to

$$
\begin{array}{ll}
\frac{\partial \vec{u}}{\partial t}+\vec{u} \cdot \nabla \vec{u}-\nu \nabla^{2} \vec{u}+g \nabla \eta+\frac{c_{b}+c_{t}(d)}{H}\|\vec{u}\| \vec{u}=0, \\
\frac{\partial \eta}{\partial t}+\nabla \cdot(H \vec{u})=0, & \forall \vec{x} \text { in } \Omega_{\text {farm }}, \\
0 \leq d(\vec{x}) \leq \bar{d}(\vec{x}) \quad \forall \vec{x} \text { in } \Omega \backslash \Omega_{\text {farm }} .
\end{array}
$$


The first two constraint equations are the shallow-water equations (5). The final two constraints ensure that the turbine density is positive, is limited by an upper bound in the farm area, and is zero outside the farm area.

Some remarks can be made about the optimisation problem (11):

- The original goal quantity (3) computes the profit over the farm's lifetime and can be measured in, for instance, US dollars. In contrast, the rescaled goal quantity in (11) is measured in units of power. The rescaled goal quantity should be interpreted as the part of the farm's average power extraction which contributes to the farm's profit. Hence, if the goal quantity is positive the farm produces a positive return, otherwise a negative. In other words, $C /(L I k)$ is the average amount of power per turbine that needs to be produced in order for the farm to break even.

- The optimal turbine density function $d$ can be used to determine the best turbine locations in the farm. It can also be used to estimate the optimal number of turbines with equation (2). This is a distinct advantage over approaches where turbines are resolved individually and the inclusion of the number of turbines as part of the optimisation results in a mixed integer problem which are generally harder to solve and more costly (Culley et al. 2014).

- The first term of the goal quantity evaluates the farm's average power production over its entire lifetime. In order to limit the computational expense this term can be estimated by computing the average power production over a smaller time window, for example one tidal cycle. To even further reduce the computational expense, a steady state solution at a representative time can be used.

- Due to the non-negativity constraint placed upon $d$, the goal quantity of (11) can be reformulated as:

$$
\frac{1}{L}\left(E(\vec{u}, d)-\frac{C}{I k} \int_{\Omega}|d(\vec{x})| \mathrm{d} \vec{x}\right) .
$$

In other words, the cost term can be identified as a scaled $L^{1}$-norm of the control function. In an optimisation context, such a term is called $L^{1}$ regularisation. Generally in optimisation, regularisation terms are used to promote optimal solutions with certain properties. For example in this case, it is used to promote farm designs with minimal cost. In addition, the $L^{1}$-regularisation has another property: it promotes sparse farm designs, that is, designs where the turbine density either vanishes or reaches its maximum value. This property is desirable for example to avoid high cabling costs (Culley et al. 2016). Additionally, it simplifies the translation of the optimal turbine density into the actual positioning of individual turbines, because areas with maximum density can simply be packed with turbines according to the minimum distance constraint. 
From a computational viewpoint, $L^{1}$-regularisation is challenging due to its non-differentiability at 0 which requires special treatment in the optimisation algorithm. However, this problem is avoided here thanks to the positivity constraint for $d$, which means that standard algorithms can be applied directly.

- The set of feasible farm designs (that is the set of turbine density functions that satisfy the last two inequality constraints in problem (11)) is convex. This convexity property is an important requirement for gradientbased optimisation methods to guarantee convergence. In particular, it is convex for any farm geometry (including disconnected and non-convex geometries), turbine constraints and minimum distance constraints between turbines. This is an advantage over the formulation in Funke et al. (2014), where the farm geometry or constraints can result in a non-convex feasible design set.

\subsection{Estimation of cost coefficient}

The value $C /(L I k)$ in $(11)$ can be estimated as follows. Consider a model turbine with a total energy extraction $E_{\text {turbine }}$ from the flow over its lifetime and with a profit margin $m$. The definition of profit margin yields:

$$
\begin{aligned}
m & =\frac{\text { Revenue }- \text { Cost }}{\text { Revenue }}=\frac{I k E_{\text {turbine }}-C}{I k E_{\text {turbine }}} \\
\Rightarrow \quad \frac{C}{L I k} & =(1-m) \frac{E_{\text {turbine }}}{L} .
\end{aligned}
$$

The second factor on the right hand side describes the average power production of the model turbine over the period $L$. For a simplified sinusoidal tidal cycle with a peak velocity magnitude $u_{\text {peak }}$ and a peak power production $P_{\text {peak }}$, the average power production can be estimated as $0.42 P_{\text {peak }}$. Using $(9)$, substituting (8) for the density function corresponding to a single turbine, i.e. $\int_{\Omega} d(\vec{x}) \mathrm{d} \vec{x}=1$, we get

$$
P_{\text {peak }}=\rho \int_{\Omega} \frac{1}{2} C_{t} A_{t} d(\vec{x}) u_{\text {peak }}^{3} \mathrm{~d} \vec{x}=\frac{1}{2} C_{t} A_{t} \rho u_{\text {peak }}^{3} .
$$

This yields the formula

$$
\frac{C}{L I k}=\frac{0.42}{2} C_{T} A_{T}(1-m) \rho u_{\mathrm{peak}}^{3} .
$$

Note that the right hand side only depends indirectly on the turbine efficiency $k$ and its lifetime $L$, through the estimated profit margin $m$. For a constant flow (instead of a sinusoidal one), equation (12) can be used without the 0.42 factor. 


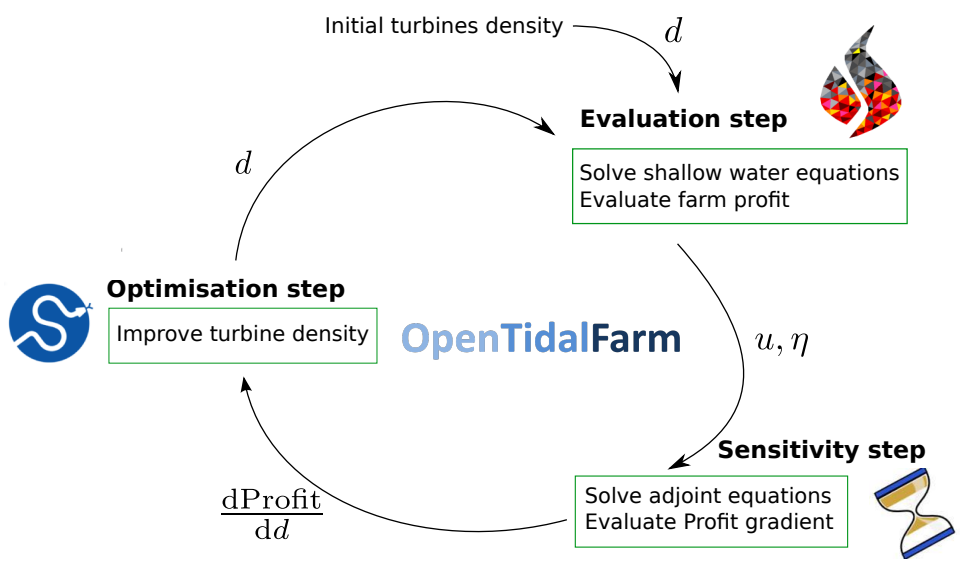

Figure 2: The optimisation loop of OpenTidalFarm and the software it relies on. OpenTidalFarm uses the finite element framework FEniCS (Logg et al., 2012) to assemble and solve the shallow water and adjoint shallow water equations (Funke et al., 2014). The adjoint shallow water equations are automatically derived by dolfin-adjoint and its optimisation capabilities (Farrell et al., 2013; Funke, 2013). We employ the L-BFGS-B method to solve the optimisation problem (Byrd) et al., 1995, Zhu et al., 1997). Each optimisation iteration consists of one (or multiple if a line search is required) solution of the shallow water equations to evaluate the farm profit, and one solution of the associated adjoint equation to compute the discretely consistent profit gradient. By combining gradient-based optimisation with the adjoint approach, the required optimisation iterations are practically independent of the size of the turbine farm.

\section{Numerical solution}

We solve problem (11) by discretising the shallow water equations with the finite element method and by solving the resulting discrete optimisation problem with a gradient-based optimisation method. The gradient of the goal quantity, that is the derivative of the profit function with respect to the turbine density function, is computed with the adjoint equations. The iteration loop of the optimisation is visualised in figure 2. The implementation is available as part of OpenTidalFarm (opentidalfarm.org), an open-source software package for tidal resource assessment and turbine farm design optimisation.

\subsection{Spatial and temporal discretisation}

We follow Funke et al. (2014) to spatially discretise the shallow water equations with a second-order finite element scheme. Multiplying the two equations making up the shallow water system by test functions $\vec{\Psi}$ and $\Phi$ respectively, integrating over the domain and integrating the viscosity and divergence terms by parts yields the weak formulation of the shallow water equations: find 
$(\vec{u}, \eta) \in V \times Q$ such that for all $(\vec{\Psi}, \Phi) \in V \times Q$ :

$$
\begin{gathered}
\left\langle\frac{\partial \vec{u}}{\partial t}, \vec{\Psi}\right\rangle_{\Omega}+\langle\vec{u} \cdot \nabla \vec{u}, \vec{\Psi}\rangle_{\Omega}+\nu\langle\nabla \vec{u}, \nabla \vec{\Psi}\rangle_{\Omega} \\
+g\langle\nabla \eta, \vec{\Psi}\rangle_{\Omega}+\left\langle\frac{c_{b}+c_{t}(d)}{H}\|\vec{u}\| \vec{u}, \vec{\Psi}\right\rangle_{\Omega}=0 \\
\left\langle\frac{\partial \eta}{\partial t}, \Phi\right\rangle_{\Omega}-\langle H \vec{u}, \nabla \Phi\rangle_{\Omega}+\langle H \vec{u} \cdot \vec{n}, \Phi\rangle_{\partial \Omega \backslash \partial \Omega_{N}}=0 .
\end{gathered}
$$

Here $\langle\cdot, \cdot\rangle$ denotes the $L^{2}$ inner product and $\vec{n}$ is the unit outward facing normal vector on the boundary $\partial \Omega$. For these equations it is natural to choose the Hilbert spaces $H^{1}(\Omega)^{2}$ and $H^{1}(\Omega)$ for $V$ and $Q$, respectively.

Dirichlet boundary conditions for the velocity and free surface are imposed by restricting the function spaces $V$ and $Q$ to functions that satisfy the boundary conditions, and by using test functions that vanish on the boundary. The free slip boundary conditions $\vec{u} \cdot \vec{n}=0$ on $\partial \Omega_{N}$ are enforced by omitting the surface integral on $\partial \Omega_{N}$.

The discrete problem is obtained by replacing the function spaces with finitedimensional subspaces $V_{h} \subset V, Q_{h} \subset Q$. For their construction one first creates a triangulation of the domain. $V_{h}$ is then chosen as continuous, piecewise quadratic functions and $Q_{h}$ as the space of continuous, piecewise linear functions, that is the classical Taylor-Hood $\left(P_{2}-P_{1}\right)$ finite element pair. The turbine density function and the associated bottom friction are represented by continuous, piecewise linear functions.

When solving a steady state problem, the time derivative terms are ignored in equations (13). In the transient case, equations (13) are discretised in time using the backward Euler scheme. Denoting time steps as superscripts, the discrete shallow water equations are: find $(\vec{u}, \eta) \in V_{h} \times Q_{h}$ such that for all $(\vec{\Psi}, \Phi) \in V_{h} \times Q_{h}:$

$$
\begin{gathered}
\left\langle\frac{\vec{u}^{n}-\vec{u}^{n-1}}{\Delta t}, \vec{\Psi}\right\rangle_{\Omega}+\left\langle\vec{u}^{n} \cdot \nabla \vec{u}^{n}, \vec{\Psi}\right\rangle_{\Omega}+\nu\left\langle\nabla \vec{u}^{n}, \nabla \vec{\Psi}\right\rangle_{\Omega} \\
+g\left\langle\nabla \eta^{n}, \vec{\Psi}\right\rangle_{\Omega}+\left\langle\frac{c_{b}+c_{t}(d)}{H^{n}}\left\|\vec{u}^{n}\right\| \vec{u}^{n}, \vec{\Psi}\right\rangle_{\Omega}=0 \\
\left\langle\frac{\eta^{n}-\eta^{n-1}}{\Delta t}, \Phi\right\rangle_{\Omega}-\left\langle H^{n} \vec{u}^{n}, \nabla \Phi\right\rangle_{\Omega}+\left\langle H^{n} \vec{u}^{n} \cdot \vec{n}, \Phi\right\rangle_{\partial \Omega \backslash \partial \Omega_{N}}=0 .
\end{gathered}
$$

\subsection{Optimisation method}

The non-discretised problem (11) is an infinite-dimensional optimisation problem in the sense that the turbine density function, the control variable, lives in an infinite-dimensional space. The dimension of the corresponding discrete optimisation problem, based on the discretised system (14), depends on the mesh and the finite element choice. For practically relevant setups, this results in a large-scale optimisation problem; for instance, the multi-farm design optimisation in section 8 has 161, 763 control dimensions. Considering that each 
profit evaluation requires solving the shallow water equations, the solution of such optimisation problems is only feasible with optimisation methods whose iteration number requirements do not depend on the dimension of the control space and which compute the solution in a small (e.g. in the order of 100 or less) number of iterations.

Optimisation methods can be classified into derivative-free and derivativebased algorithms. Derivative-free methods, such as genetic algorithms, simulatedannealing or Powell's conjugate direction method, rely purely on evaluating the goal quantity at different points in control space. Derivative-free methods are easily applicable because the hydrodynamic solver can be treated as a blackbox. However, they typically require a large number of iterations and scale unfavourably with the dimension of the control space. Hence these methods become quickly infeasible for the problem considered here.

In contrast, derivative-based optimisation methods use both evaluation of the goal quantity and its derivative with respect to the control variables. As such, a solver for hydrodynamic equations alone is not sufficient, because the efficient computation of derivatives requires a solver for the corresponding adjoint equations (see section 5.3). Derivative-based methods are very efficient for large-scale problems, and often require only a small number of iterations, independent of the problem discretisation. One potential drawback of derivative based methods is that they only guarantee local convergence, that is they might not be able to find the global optimal solution. However, in our experience this is mostly a problem if the feasible set of control functions is non-convex, which is avoided with the proposed problem formulation here (see last remark in section (4)). In this paper we use the limited-memory BFGS method with bound constraints (L-BFGS-B) to solve the optimisation problems (Byrd et al. 1995, Zhu et al. 1997).

\subsection{The adjoint equations}

The L-BFGS-B method requires the gradient of the profit function with respect to the turbine density function. However, the profit function, 10 or (11), depends on the turbine density both directly as well as indirectly through the dependence of the velocity on the turbine density. One could approximate the gradient through a finite difference like approach, but this would require as many profit evaluations as there are unknowns in the turbine friction representation. Each evaluation requires solving the shallow water equations to provide $u$ for a given $d$, making this only feasible for small problems.

In contrast, the adjoint approach computes the gradient by solving only a single linear partial differential equation system, termed the adjoint equations corresponding to the underlying shallow water equations. This means that the computational cost becomes practically independent of the problem size. The gradient is computed in the following three steps:

1. Solve the shallow water equations for $\vec{u}, \eta$ (section 5.1). Note that this computation needs to be performed regardless in order to evaluate the farm profit. 
2. Solve the adjoint shallow water equations for the adjoint velocity $\vec{\lambda}_{u}$ and adjoint free-surface displacement $\lambda_{\eta}$ :

$$
\begin{aligned}
-\frac{\partial \vec{\lambda}_{u}}{\partial t}+ & (\nabla \vec{u})^{*} \vec{\lambda}_{u}-(\nabla \cdot \vec{u}) \vec{\lambda}_{u}-\vec{u} \cdot \nabla \vec{\lambda}_{u}-\nu \nabla^{2} \vec{\lambda}_{u} \\
& -H \nabla \lambda_{\eta}+\frac{c_{b}+c_{t}(d)}{H}\left(\|\vec{u}\| \vec{\lambda}_{u}+\frac{\vec{u} \cdot \vec{\lambda}}{\|\vec{u}\|} \vec{u}\right)=\frac{\partial \text { Profit }^{*}}{\partial \vec{u}} \\
- & \frac{\partial \lambda_{\eta}}{\partial t}-g \nabla \cdot \vec{\lambda}_{u}-\nabla \lambda_{\eta} \cdot \vec{u}-\frac{c_{b}+c_{t}(d)}{H^{2}}\|\vec{u}\| \vec{u}=0 .
\end{aligned}
$$

For brevity we refer to Funke (2013, appendix C) for the derivation of these equations. Note that the adjoint equations are linear even though the shallow water equations are non-linear, and that they are solved backward in time with a zero final-time condition. The Dirichlet boundary conditions are the homogenised boundary conditions of the shallow water equations.

In order to obtain a discretely consistent gradient (such that the gradient is the exact derivative of the discrete model), the adjoint equations must use the same discretisation scheme as the shallow water equations. The discrete adjoint shallow water equations are thus: find $\left(\vec{\lambda}_{u}, \lambda_{\eta}\right) \in V_{h} \times Q_{h}$ such that for all $(\vec{\Psi}, \Phi) \in V_{h} \times Q_{h}$ :

$$
\begin{gathered}
\left\langle\frac{\vec{\lambda}_{u}^{n}-\vec{\lambda}_{u}^{n+1}}{\Delta t}, \vec{\Psi}\right\rangle_{\Omega}+\left\langle\vec{\lambda}_{u}^{n}, \vec{\Psi} \cdot \nabla \vec{u}^{n}\right\rangle_{\Omega}+\left\langle\vec{\lambda}_{u}^{n}, \vec{u}^{n} \cdot \nabla \vec{\Psi}\right\rangle_{\Omega} \\
+\nu\left\langle\nabla \vec{\lambda}_{u}^{n}, \nabla \vec{\Psi}\right\rangle-\left\langle H^{n} \nabla \lambda_{\eta}^{n}, \vec{\Psi}\right\rangle_{\Omega}+\left\langle H^{n} \lambda_{\eta}^{n}, \vec{\Psi} \cdot \vec{n}\right\rangle_{\partial \Omega \backslash \partial \Omega_{N}} \\
+\left\langle\frac{c_{b}+c_{t}(d)}{H^{n}}\left(\left\|\vec{u}^{n}\right\| \vec{\Psi}+\frac{\vec{u}^{n} \cdot \vec{\Psi}}{\left\|\vec{u}^{n}\right\|} \vec{u}^{n}\right), \vec{\lambda}_{u}^{n}\right\rangle_{\Omega}=\left\langle\frac{\partial \text { Profit }}{\partial \vec{u}^{n}}, \vec{\Psi}\right\rangle_{\Omega} \\
\left\langle\frac{\lambda_{\eta}^{n}-\lambda_{\eta}^{n+1}}{\Delta t}, \Phi\right\rangle_{\Omega}+g\left\langle\vec{\lambda}_{u}^{n}, \nabla \Phi\right\rangle_{\Omega}-\left\langle\nabla \lambda_{\eta}^{n}, \Phi \vec{u}^{n}\right\rangle_{\Omega} \\
+\left\langle\lambda_{\eta}^{n}, \Phi \vec{u}^{n} \cdot \vec{n}\right\rangle_{\partial \Omega \backslash \partial \Omega_{N}}-\left\langle\frac{c_{b}+c_{t}(d)}{\left(H^{n}\right)^{2}} \Phi\left\|\vec{u}^{n}\right\| \vec{u}^{n}, \vec{\lambda}_{u}^{n}\right\rangle=0 .
\end{gathered}
$$

Note that the adjoint equations depend on the velocity solution at all timesteps. Here, we apply an "all-in-memory" approach, where we store all velocity solutions from step 1 in memory. For large-scale problems where this becomes a bottleneck, a disk-based check-pointing scheme could be used.

3. Evaluate the gradient of the goal quantity with

$$
\frac{\mathrm{dProfit}}{\mathrm{d} d}=-\sum_{n}\left(\frac{C_{T} A_{T}}{2 H^{n}}\left\|\vec{u}^{n}\right\| \vec{u}^{n} \cdot \vec{\lambda}_{u}^{n}\right)+\frac{\partial \text { Profit }}{\partial d} .
$$




\begin{tabular}{llrl}
\hline Parameter & Symbol & \multicolumn{2}{c}{ Value } \\
\hline Water density & $\rho$ & 1000 & $\mathrm{~kg} \mathrm{~m}^{-3}$ \\
Viscosity & $\nu$ & 0.5 & $\mathrm{~m}^{2} \mathrm{~s}^{-1}$ \\
Water depth at rest & $h$ & 50 & $\mathrm{~m}$ \\
Gravity & $g$ & 9.81 & $\mathrm{~m} \mathrm{~s}^{-2}$ \\
Natural bottom friction & $c_{b}$ & 0.0025 & \\
Turbine diameter & & 20 & $\mathrm{~m}$ \\
Minimum distance between turbines & $D_{\min }$ & 40 & $\mathrm{~m}$ \\
Maximum turbine density & $\bar{d}$ & $6.25 \cdot 10^{-4}$ & $\mathrm{~m}^{-2}$ \\
Thrust coefficient & $C_{T}$ & 0.6 & \\
Turbine cross section & $A_{T}$ & 314.15 & $\mathrm{~m}^{2}$ \\
Profit margin & $m$ & 40 & $\%$ \\
Cost coefficient (break even power) & $C /(L I k)$ & 452.39 & $\mathrm{~kW}$ \\
\hline
\end{tabular}

Table 1: Parameters for the continuous versus discrete farm optimisation comparison.

\section{Continuous versus discrete farm optimisation}

Funke et al. (2014) proposed a discrete farm layout optimisation approach with the objective to find the individual turbine locations that maximise the farm's power production (so-called turbine micro-siting). The drag force exerted by a turbine was represented via an increased bottom drag in the shallow water model, but in contrast to the continuous approach proposed here the turbines were explicitly resolved in the numerical model/mesh. More precisely, the farm induced drag consisted of a sum of individual friction 'bump' functions, each representing one tidal turbine. In that case the control parameters for the optimisation were the locations of the bump centres, that is the locations of the individual turbines.

Given the similar objectives of this work, it is natural to ask how the discrete and continuous approaches compare. In particular, our goal is to address the following questions: Do the discrete and continuous approaches yield similar optimal farm configurations and profit predictions? Are the predicted farm performances comparable? Is the optimal number of turbines computed by the continuous optimisation consistent with the discrete approach?

In an attempt to address these we applied both strategies to the same idealised problem. The domain considered is a square basin with sides of $4 \mathrm{~km}$, and $50 \mathrm{~m}$ depth. The farm area is restricted to a square with sides of $1 \mathrm{~km}$ located in the centre the domain. For the discrete turbine optimisation, the turbine positions are restricted to this same area. The boundary conditions are an inlet flow of $2 \mathrm{~m} \mathrm{~s}^{-1}$ on the west boundary, a free surface elevation $\eta=0$ on the east boundary and free-slip conditions on the north and south boundaries. The remaining parameters are listed in table 1. With this setup, the flow in the channel is steady, which means that a steady-state shallow water solver could be used. 


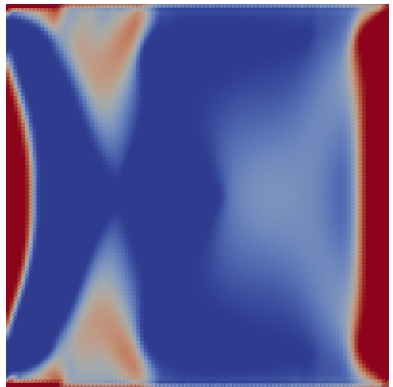

(a) Continuous turbine approach.

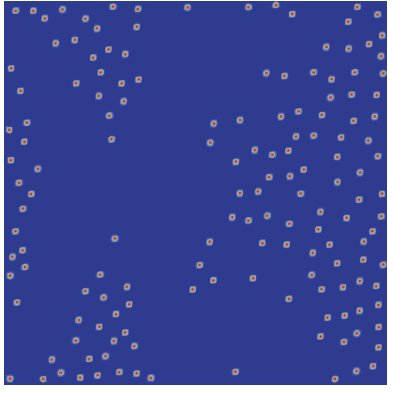

(b) Discrete layout from ( continuous approach.

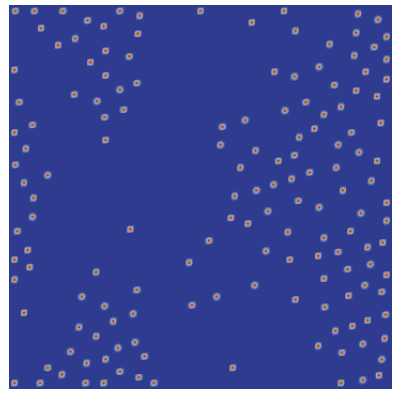

(c) Discrete turbine approach.

Figure 3: (a) Optimal turbine density field for the continuous versus discrete farm optimisation comparison. Only the farm area is shown. Blue indicates a farm density of 0 . Red indicates a farm density of the upper bound $\bar{d}$. The integrated turbine density field corresponds to 152 turbines. (b) Positions of the individual turbines derived from the optimal turbine density function using algorithm 1 with $N=152$. (c) Turbine positions after applying the discrete optimisation approach using (b) as an initial layout.

\subsection{Continuous turbine optimisation}

We solved the continuous farm design problem with the steady-state shallow water equations. The steady-state solution allows us to compute $E / L$ directly as the power extracted according to (9). The cost coefficient is calculated using equation (12) without the 0.42 factor and a value of $u_{\text {peak }}$ that is equal to the inlet velocity, see table 1 . The computational mesh consisted of 40,590 elements with a typical element size of $10 \mathrm{~m}$ in the farm area and $100 \mathrm{~m}$ elsewhere.

The problem was discretised and solved as discussed in section 5. The nonlinear shallow water problem was solved with Newton's method and a direct linear solver for the linear sub-problems and the adjoint problem. After 274 optimisation iterations the relative increase in the goal quantity per iteration dropped below $2.2 \times 10^{-6}$ at which point the optimisation algorithm terminated.

The resulting optimal turbine density function is shown in figure $3 \mathrm{a}$. The optimal number of turbines is determined with equation (2) and yields $N=152$. The power generated is $89.2 \mathrm{MW}$, or an average power production of $586 \mathrm{~kW}$ per turbine. The cost of the farm, measured as extracted power needed to break even for the optimal number of turbines, is 68.8 MW. The difference, 20.4 MW is the profit measured in units of power.

\subsection{Conversion of the turbine density function into turbines positions}

Given the turbine density field obtained by the optimisation process, we can derive individual positions for the turbines as they are represented in the discrete approach via the following algorithm:

Algorithm 1. Conversion of turbine density function to turbine locations 
1. Determine the number of turbines, $N$, using equation (2).

2. Repeat until $N$ turbines have been deployed:

(a) Pick a random point $\vec{x}$ in the domain.

(b) Place a turbine at $\vec{x}$ with probability $d(\vec{x}) / \max _{\Omega} \bar{d}$ if the minimum distance to all other turbines is at least $D_{\min }$.

Note that this algorithm only deploys turbines where $\bar{d}>0$ and always generates layouts that satisfy the minimum distance constraints between the turbines. The result of the algorithm applied to the test problem is shown in figure 3b. Comparing figures $3 \mathrm{a}$ and $3 \mathrm{~b}$ indicates that the algorithm is able to capture most of the spatial structure from the turbine density function.

Next, we investigated how the continuous and discrete turbine representations impact the hydrodynamic flow, and how much consistency there is in the predicted power and profit. We configured the discrete turbine approach with the same settings as the continuous turbine model. The turbine induced friction was set to a sum of bump functions centred at the positions obtained from algorithm 1. The bump diameter was set to the turbine diameter and the bump amplitude was chosen such the integrated friction of the discrete and continuous farm are identical. Since the discrete approach has to resolve the bump functions, the mesh needed to be refined to $5 \mathrm{~m}$ resolution over the farm area.

\subsection{Turbine density function as an initial guess for discrete micro-siting}

The turbine locations obtained with algorithm 1 can additionally be used to provide an initial guess for a discrete farm optimisation. If the continuous approach generates good turbine concentrations, and consequently potential locations for individual turbines, one perhaps would expect only marginal improvements from a subsequent optimisation with the discrete approach. A consequence of this could also be that fewer iterations of the more costly discrete optimisation may be needed. Hence, a combined 'continuous $\rightarrow$ discrete' process may provide broadly the same, or potentially better, answer with a smaller overall computational cost compared to using the discrete approach in isolation.

To test this, we performed two numerical experiments based on the idealised problem considered earlier in this section. Based on the value of $N$ obtained during the continuous optimisation above, the first experiment optimised the layout of $N=152$ turbines with the discrete approach, starting from a regular non-staggered grid layout. In this case, the initial power production was 41.4 MW, which the discrete optimisation increased to 84.6 MW (the cost term remains constant as the number of turbines is constant).

The second experiment used the turbine positions derived from the continuous approach as an initial guess (as shown in figure 3b). In this case, the initial power extraction was $79.4 \mathrm{MW}$, i.e. just using the very simple algorithm 1 provides a substantial improvement over a grid based initial layout, with a value within approximately $12 \%$ of that predicted by the continuous approach (89.2 MW). Following discrete optimisation from this initial guess the optimised power extraction was $84.5 \mathrm{MW}$. The optimisation performs only small adjustments to the turbine positions and the final layout is shown in figure $3 \mathrm{c}$ 


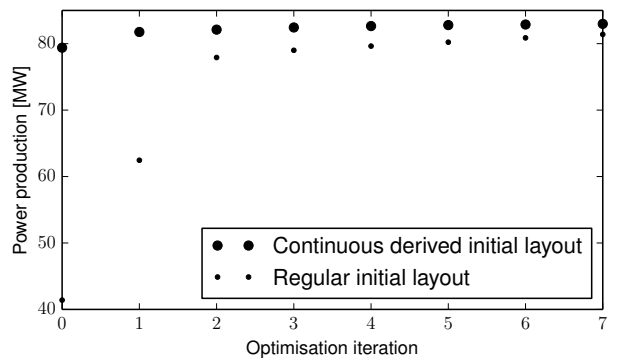

Figure 4: The power production during the first eight optimisation iterations for two different initial layouts.

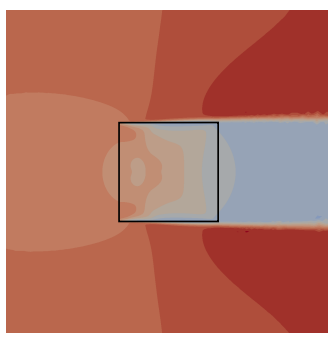

(a)

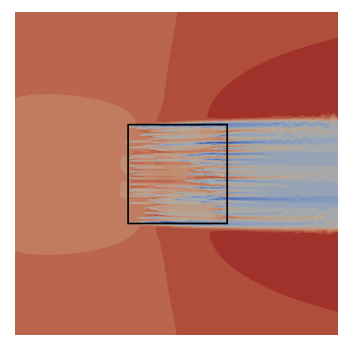

(b)

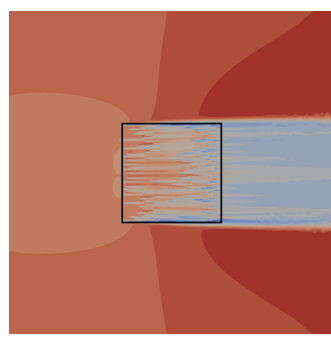

(c)

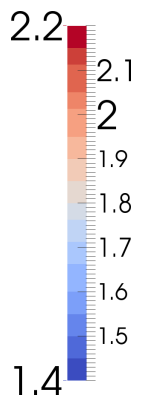

2.1

Figure 5: Velocity magnitudes $\left(\mathrm{m} \mathrm{s}^{-1}\right)$ in the presence of (a) the optimised continuous turbine farm, (b) the discrete turbine farm converted from the continuous optimisation result using algorithm 1, and (c) the discrete farm after further optimising using the discrete approach.

Figure 4 shows an iteration plot of the first eight optimisation iterations for both experiments: the initial guess derived from the continuous solution starts already close to the optimal power production, and reaches the optimum practically after one iteration. The optimisation with the initial regular layout converges to essentially the same optimal power production, but requires more iterations.

These results suggest that the continuous approach may be directly used to provide a valuable farm design in its own right. And further, if computationally feasible, the locations can be further refined with a few iterations of the more costly discrete optimisation.

\subsection{Comparison of hydrodynamic solutions}

The hydrodynamic solutions for both turbine representations are compared in figure 5. Figure 5a shows the velocity magnitude in the presence of the optimised continuous turbine farm, and figure $5 \mathrm{~b}$ the solution with the discrete farm derived directly from the continuous solution. Starting from this layout 


\begin{tabular}{ccccc}
\multicolumn{2}{c}{ Experiment } & Power $(\mathrm{MW})$ & Cost $(\mathrm{MW})$ & Profit $(\mathrm{MW})$ \\
\hline \multicolumn{2}{c}{ continuous } & $\mathbf{8 9 . 2 1}$ & $\mathbf{6 8 . 8 1}$ & $\mathbf{2 0 . 3 9}$ \\
\hline discrete & $\mathrm{N}=102$ & 59.89 & 46.18 & 13.71 \\
discrete & $\mathrm{N}=127$ & 72.61 & 57.49 & 15.12 \\
discrete & $\mathrm{N}=\mathbf{1 5 2}$ & $\mathbf{8 4 . 4 5}$ & $\mathbf{6 8 . 8 1}$ & $\mathbf{1 5 . 6 4}$ \\
discrete & $\mathrm{N}=177$ & 95.03 & 80.12 & 14.91 \\
discrete & $\mathrm{N}=202$ & 104.20 & 91.44 & 12.76
\end{tabular}

Table 2: Results from the continuous versus discrete farm optimisation comparison. The continuous approach predicts that 152 turbines yields the maximum profit. This is confirmed by running a set of discrete optimisation runs with varying number of turbines. The continuous approach results in a slightly higher predicted power extraction because its control space has more degrees of freedom than the discrete approach.

as an initial guess, figure $5 \mathrm{c}$ gives the flow solution after applying the discrete approach to further optimise this farm. As expected, the discrete approach shows a much more detailed flow pattern inside the farm area, but with a broadly consistent build up of a farm deficit within the farm area. In addition the patterns of farm wake and bypass flow also match very well with the pattern in the continuous approach. Going from figure $5 \mathrm{~b}$ to $5 \mathrm{c}$, the discrete solution gets even closer to the continuous solution in figure $5 \mathrm{a}$ as the discrete farm is further optimised. This indicates that the continuous approach gives a good approximation of the large scale flow pattern when optimising turbine arrays.

\subsection{Optimal number of turbines}

As already stated, a significant strength of the continuous approach is that it also predicts the optimal number of turbines for a farm site. We performed a series of experiments to check if this number is also considered to be optimal in the discrete approach.

First, we determined the optimal number of turbines $N=152$ with equation (2). Then, we applied algorithm 1 to generate initial layouts for the discrete approach with $N, N \pm 25$ and $N \pm 50$ turbines, and performed a discrete layout optimisation for each of them. The results are listed in table 2 . The table shows that the discrete optimisation with 152 turbines produces the highest profit. Therefore the continuous approach indeed correctly predicted the optimal number of turbines (to within a tolerance of around $16 \%$ based on the numbers considered in this particular case).

\section{Setup and validation of the Pentland Firth forward model}

The examples in sections 8 and 9 to follow are based on a forward model of the Pentland Firth, a strait between the Scottish mainland and the Orkney Islands. It is an area of significant interest for the tidal renewable industry with several leasing areas assigned by the Crown Estate. In one of these, in the Inner 


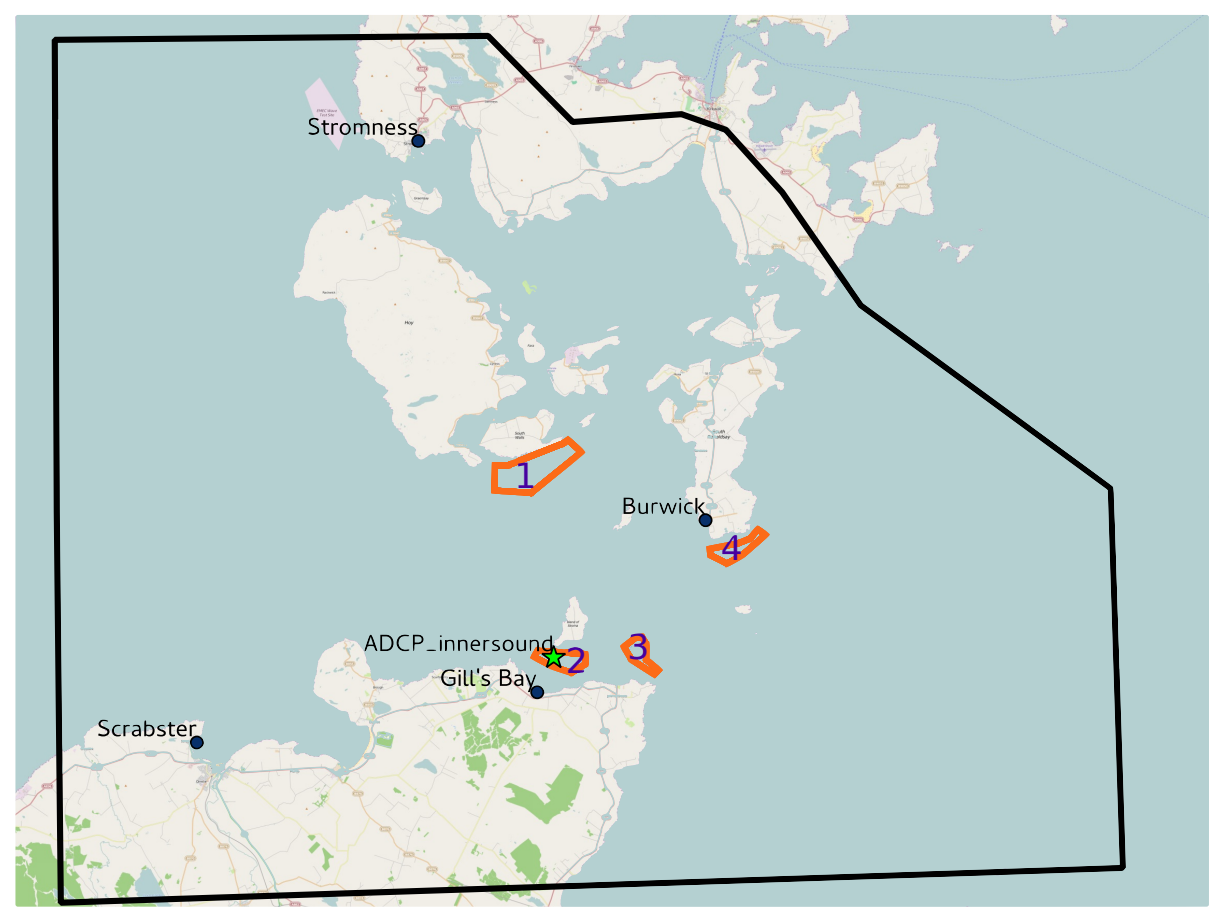

Figure 6: The Pentland Firth between the Scottish mainland and the Orkney Islands. The outline of the modelled area is indicated in black. For the model validation, data from four tide gauge locations at Scrabster, Stromness, Gill's Bay and Burwick, and from an ADCP in the Inner Sound have been used. The farm areas optimised in section 8.2 are indicated and numbered: 1) Cantick Head, 2) Inner Sound, 3) Ness of Duncansby, and 4) Brough Ness. 
Sound to the south of the island of Stroma, the construction and deployment of an initial small number of turbines has been approved and should be underway soon. This initial deployment is hoped to be scaled up to a large farm with a power production of $398 \mathrm{MW}$.

The model of the Pentland Firth covers a domain shown in figure 6. Two different meshes were employed both with edge lengths varying between $350 \mathrm{~m}$ and $25 \mathrm{~m}$. The smallest edge lengths were only employed inside the designated farm areas, with resolution smoothly coarsening outside of these. The mesh used in section 8 consists of 321,224 elements and defines four farm areas which are roughly based on four different lease sites assigned by the Crown Estate (The Crown Estate, 2013): 1) Cantick Head, 2) Inner Sound, 3) Ness of Duncansby, and 4) Brough Ness. The mesh used in section 9 only incorporates the Inner Sound site (farm 2) as a high resolution area, and thus consists of considerably fewer elements $(115,022)$. Outside of the farm area(s) the mesh resolutions are chosen in the same manner. The validation shown here is based on the mesh from section 9. As expected, the results with the mesh from section 8 were found to be very similar.

It should be noted that the chosen domain is smaller than the recommendations in Adcock et al. (2011), which suggests the open boundaries should be a $100 \mathrm{~km}$ to the east of Muckle Skerry at eastern exit of the Pentland Firth, and $200 \mathrm{~km}$ to the west of the western entrance. Our choice was a compromise to limit the computational expense. We did however check for differences in flow velocities at the open boundaries between the base case without turbines and a case with all four farms present (see section 8), and found the differences to be small (less than $0.02 \mathrm{~ms}^{-1}$ at any point along the open boundaries for the entire simulation time).

The bathymetry used in both setups is obtained from four different sources: 1) the global bathymetry data set GEBCO_08 (British Oceanographic Data Centre (BODC), 2010) with a resolution of $30 \operatorname{arc-sec}(\approx 900 \mathrm{~m}), 2)$ Digimap (Edina Digimap service, 2014$)$ which gives a resolution of 1 arc-seconds $(\approx 30$ $\mathrm{m}$ ) near the UK coast, 3) bathymetry data from the Scottish Goverment (2009) at $2 \mathrm{~m}$ resolution covering most of the Pentland Firth, but missing parts of the Inner Sound near the coast, and 4) bathymetry data obtained from MeyGen, also at $2 \mathrm{~m}$ resolution but covering a larger part of the Inner Sound. These four sources were combined to obtain the most reliable data in each area. The result was then projected onto the mesh and smoothed.

The models are forced with free surface elevation boundary conditions reconstructed from tidal harmonics based on a global tidal solution using the OSU Tidal Prediction Software (OTPS, Egbert and Erofeeva (2010)). At the coastal boundaries a no-slip condition was applied. No wetting and drying scheme was applied here, but a minimum depth of $10 \mathrm{~m}$ constraint was applied to the bathymetry used in order to avoid negative water depths. The models use a fixed eddy viscosity of $10 \mathrm{~m}^{2} \mathrm{~s}^{-1}$ everywhere, except for a band of $1 \mathrm{~km}$ around the open boundaries where an increased viscosity of $100 \mathrm{~m}^{2} \mathrm{~s}^{-1}$ is applied to avoid instabilities typically arising at open boundaries in nonlinear models. For time integration the backward Euler method was applied with a time step of 


\begin{tabular}{l|rrrrrrrrr} 
& \multicolumn{3}{|c}{ M2 } & \multicolumn{2}{c}{ S2 } & \multicolumn{3}{c}{ K1 } & \multicolumn{2}{c}{ O1 } \\
& amp & pha & amp & pha & amp & pha & amp & pha \\
\hline Charted & & & & & & & & \\
Scrabster & 1.35 & 247 & 0.51 & 280 & 0.14 & 144 & 0.10 & 349 \\
Stromness & 0.89 & 270 & 0.35 & 303 & 0.11 & 148 & 0.10 & 5 \\
Gill's Bay & 1.12 & 268 & 0.41 & 300 & 0.13 & 151 & 0.11 & 360 \\
Burwick & 0.88 & 287 & 0.35 & 322 & 0.14 & 158 & 0.12 & 17 \\
\hline Model $\left(c_{b}=0.0025\right)$ & & & & & & & & \\
Scrabster & 1.27 & 247 & 0.42 & 290 & 0.14 & 157 & 0.11 & 359 \\
Stromness & 0.94 & 270 & 0.31 & 313 & 0.13 & 167 & 0.11 & 14 \\
Gill's Bay & 0.96 & 276 & 0.31 & 318 & 0.13 & 166 & 0.11 & 11 \\
Burwick & 0.86 & 291 & 0.27 & 335 & 0.13 & 167 & 0.11 & 15 \\
\hline Model $\left(c_{b}=0.003\right)$ & & & & & & & & \\
Scrabster & 1.27 & 247 & 0.42 & 290 & 0.14 & 157 & 0.11 & 359 \\
Stromness & 0.95 & 270 & 0.31 & 312 & 0.13 & 167 & 0.10 & 14 \\
Gill's Bay & 0.96 & 275 & 0.31 & 318 & 0.13 & 166 & 0.11 & 12 \\
Burwick & 0.86 & 291 & 0.27 & 335 & 0.14 & 167 & 0.11 & 16 \\
\hline
\end{tabular}

Table 3

$600 \mathrm{~s}$.

For the validation of the models, the setup was run for a period of 20 days starting on the 4th of July 2011 at midnight. This period was chosen to overlap with the ADCP data available. The first day of model output was not used in the analysis to allow for the model to spin up.

At four different locations in the domain (Scrabster, Stromness, Gill's Bay and Burwick, see figure 6), the free surface solution was recorded. From these time series the four main harmonic constituents (M2, S2, K1 and O1) were derived using the T_Tide Harmonic Analysis Toolbox (Pawlowicz et al., 2002) in MATLAB. The results are shown in table 3 where they are compared with values based on measurements at these locations (UKHO, 2009). The main driver of the tidal currents through the Pentland Firth is the phase difference across the channel. It is therefore important to get the phases correct in the different locations along the channel. From the table it becomes clear that there is good agreement for the main M2 constituent and reasonable agreement for S2. These results are very similar to those presented in Easton et al. (2012) and Martin-Short et al. (2015).

For a validation of the predicted currents the measured ADCP data from a location in the Inner Sound was available. It is located inside farm area 2 which will be used in the optimisation runs in the following sections. In figure 7. we display both components of velocity for the first two days of the analysis period, comparing the model results with the ADCP measurements. The ADCP data was available at averaging intervals of $10 \mathrm{~min}$ which will have filtered out the very high frequency signal. It can however be observed that the signal still contains a lot of relatively high frequency oscillations, that are particular strong 

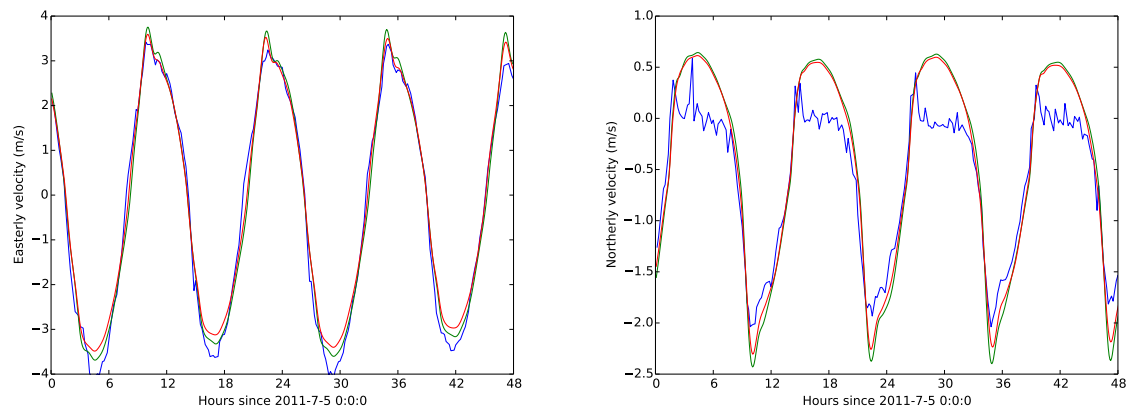

Figure 7: A comparison between both components of velocity (left figure: easterly, right figure: northerly) measured by an ADCP inside the Inner Sound farm (blue), and OpenTidalFarm model output for two values of bottom friction: $c_{b}=0.0025$ (green) and $c_{b}=0.003$ (red). The figure compares the depth average of the measured ADCP profile with the outcomes of the shallow water model.

\begin{tabular}{l|ccccccc} 
& $\begin{array}{c}\text { Speed } \\
\text { Bias Ebb } \\
(\mathrm{m} / \mathrm{s})\end{array}$ & $\begin{array}{c}\text { Speed } \\
\text { Bias Flood } \\
(\mathrm{m} / \mathrm{s})\end{array}$ & $\begin{array}{c}\text { Speed } \\
\text { RMS } \\
\text { Error } \\
(\mathrm{m} / \mathrm{s})\end{array}$ & $\begin{array}{c}\text { Speed } \\
\text { Scatter } \\
\text { Index }\end{array}$ & $\begin{array}{c}\text { Speed } \\
\text { Correla- } \\
\text { tion } \\
\text { Coefficient }\end{array}$ & $\begin{array}{c}\text { Direction } \\
\text { Bias Ebb } \\
(\mathrm{deg})\end{array}$ & $\begin{array}{c}\text { Direction } \\
\text { Bias Flood } \\
(\mathrm{deg})\end{array}$ \\
\hline$c_{b}=0.0025$ & -0.06 & 0.20 & 0.36 & 0.16 & 0.94 & -12 & -1 \\
$c_{b}=0.003$ & -0.19 & 0.13 & 0.32 & 0.14 & 0.95 & -12 & -1
\end{tabular}

Table 4: Statistics of the comparison, over 19 days, between ADCP and model outcomes for two different values of bottom friction: $c_{b}=0.0025$ and $c_{b}=0.003$. See Martin-Short et al. (2015) for definitions.

during the ebb (westward flow) period. These can be ascribed to the turbulent nature of the flow. The main differences with the model results can be seen during the same ebb period: where the model shows a consistent, small northerly component turning the mainly westward current a few degrees northward, the ADCP shows a strongly oscillatory signal with the current direction alternating around the westward direction.

In table 4 the main statistics of the comparison between ADCP and model outcomes are given. Here 19 days of simulation time are compared. The table confirms the bias of 12 degrees northward in the direction of the current, and an underprediction in speed during the ebb period that was also observed for the first two days in figure 7. Of the two values for the background bottom friction coefficient, $c_{b}=0.0025$ and $c_{b}=0.003$, that were considered, slightly better results are produced with $c_{b}=0.003$. This value was therefore chosen in the following sections. As expected the bottom friction coefficient has less of an impact on the free surface elevations as becomes clear from table 3. 


\section{Pentland Firth farm design optimisation}

\subsection{Individual farm optimisation}

We applied the continuous farm design approach separately to the four tidal farm sites in the Pentland Firth, UK (figure 6). The computational domain was equivalent to the domain discussed in section 7 . The mesh element sizes ranged between $350 \mathrm{~m}$ and $200 \mathrm{~m}$ outside the farm areas, and $25 \mathrm{~m}$ in the farm area. The simulation time spans over one tidal cycle $(12.5 \mathrm{~h})$, starting at 5:01:35am on 5 July 2011, with a 5 min time step. The initial velocity condition was taken from the simulation discussed in section 7, to avoid the need for a spinup period. The starting time was chosen, between subsequent slack tides, such that the impact of changes in friction in the farm area would have minimal impact on the current starting from the initial condition. The remaining parameters are equivalent to the ones discussed in section 7. Turbine characteristics were chosen to be typical for turbines expected to be installed in the area: a turbine diameter of $20 \mathrm{~m}$, i.e. $A_{t}=314 \mathrm{~m}^{2}$, was chosen with a fixed thrust coefficient of $C_{t}=0.6$, which is significantly lower than the optimal value of $8 / 9$ but more realistic for large scale turbines in operating conditions. For comparison a Hammerfest HS-1000 at rated speed of $2.7 \mathrm{~ms}^{-1}$ has a published (Andritz Hydro Hammerfest, 2012) rated power of $1 \mathrm{MW}$, whereas our idealized turbine produces $1.5 \mathrm{MW}$ at this speed. For simplicity we do not include rating in our calculations, but assume a constant thrust coefficient (see section 3.4 for a discussion). This means that at higher speeds we extract an unrealistic amount of power, e.g. $5 \mathrm{MW}$ at $4 \mathrm{~ms}^{-1}$.

The cost coefficient, that is the average power production per turbine to break even, was derived from 12 with a thrust coefficient $C_{T}=0.6$ and turbine cross section $A_{T}=314 \mathrm{~m}^{2}$ as mentioned, and water density $\rho=1000 \mathrm{~kg} \mathrm{~m}^{-3}$, profit margin $m=73 \%$, and peak velocity $u_{\text {peak }}=3.5 \mathrm{~m} \mathrm{~s}^{-1}$. This yields $C / L I k=452 \mathrm{~kW}$. Note that the cost coefficient is expected to have a large impact on the optimal number of turbines. The minimum distance between two turbines is set to $D_{\min }=40 \mathrm{~m}$, which results in a maximum turbine density of $\bar{d}=6.25 \times 10^{-4} \mathrm{~m}^{-2}$ in the farm.

For each farm, we terminated the optimisation after 50 optimisation iterations. On average, this required 54 shallow water and 50 adjoint shallow water solves. At this point there was no visible change in the farm design and the relative change in the profit function per iteration was always below $0.001 \%$. For testing purposes, more iterations were performed but yielded indistinguishable results.

The optimal designs for the four farms are shown in figure 8 (left images). The following key characteristics in the optimal designs can be observed:

- The optimal designs differ significantly between the farms, confirming that site-specific design optimisation is important.

- The turbines are not evenly spread across the farm, but rather concentrate around a few areas with high turbine densities. 
- A common pattern in the optimal design are barrage-like structures aligned perpendicular to the flow.

- It can be beneficial to deploy turbines along the site boundary.

The striking differences between the different sites can be explained by differences in the local flow characteristics, in particular the alignment of the farm area with the streamlines of the flow. As an example, the optimal solution for farm 2 in the Inner Sound of Stroma, seems to suggest a double row of turbines in the eastern half of the farm. The location of the first (western) of these two fences is probably guided by a principle to maximize the width over which the turbines can block the flow including the most southern streamlines that can be reached from within the farm area. The position of the second (eastern) fence is constrained by the fact that putting it further east would limit the width of the stream tube that is captured, and keeping enough distance from the first one. The middle fence is placed in such a way that the streamline furthest north is reached and the accelerated flow in the jet that comes off the tip of the island is captured. Finally, a fence on the far west of the farm area is suggested. These considerations demonstrate that for the design of a lease area, which is typically guided by various other constraints such as bathymetry and other commerical usage, a good understanding of the local tidal flow is also essential.

The profit, power, cost, and number of turbine predictions are listed in table 5 (top four rows). Note that for consistency with the next section, an additional hydrodynamic run was performed to diagnose these values, with all four optimal farm designs incorporated in the same run, even though their designs had been obtained without the presence of other farms. The design of each farm reaches its maximum profit with the deployment of hundreds of turbines. In this experiment, farm 2 is arguably the most promising, in terms of the number of turbines, the average power production, and profit. In contrast farm 1 yields a relatively small profit, despite its large site area and large number of turbines.

\subsection{Simultaneous multi-farm optimisation}

The farm design optimisations conducted so far assumed that no other farm is installed within the immediate vicinity. Since there exist only a limited number of tidal sites with strong currents, multiple site developers often plan tidal farms in relatively close proximity to each other. For the site developers it is therefore of practical importance to understand how tidal farms influence each other, and the impact of any interactions in terms of the optimal designs and profit predictions (Draper et al., 2013, 2014).

To study the influence of multiple farms, we performed another design optimisation, in which the design of all four farms were optimised simultaneously to maximise the overall profit. The setup is otherwise identical to the previous section.

The optimal design of the multi-farm optimisation is shown in figure 8 (right

column). The average profit, power, cost and optimal number of turbines are 


\begin{tabular}{l|ccccc} 
Site & $\begin{array}{c}\text { Site area } \\
\left(\mathrm{km}^{2}\right)\end{array}$ & $\begin{array}{c}\text { Avg profit } \\
(\mathrm{MW})\end{array}$ & $\begin{array}{c}\text { Avg power } \\
(\mathrm{MW})\end{array}$ & $\begin{array}{c}\text { Cost } \\
(\mathrm{MW})\end{array}$ & $\begin{array}{c}\text { Number of } \\
\text { turbines }\end{array}$ \\
\hline Farm 1 & 9.9 & $31.2(29.1)$ & $134.4(130.4)$ & $103.2(101.3)$ & $228(224)$ \\
Farm 2 & 3.1 & $195.7(180.3)$ & $316.0(293.9)$ & $120.3(113.6)$ & $266(251)$ \\
Farm 3 & 2.3 & $70.5(48.0)$ & $183.3(121.1)$ & $112.8(73.2)$ & $249(162)$ \\
Farm 4 & 2.8 & $65.5(63.8)$ & $136.9(133.2)$ & $70.4(69.4)$ & $156(153)$ \\
Total & 18.3 & $362.9(321.2)$ & $769.6(678.6)$ & $406.7(357.5)$ & $899(790)$
\end{tabular}

Table 5: Farm design optimisations results for four tidal farms in the Pentland Firth. The non-bracketed values are the results when each farm was individually optimised to maximise profit, neglecting the existence of the other farms. The values in brackets show the results when simultaneously optimising all four farms to maximise the overall profit. The discrepancy between the values indicates the risk of tidal farm developers to overpredict the farm potential when not taking into account other tidal farms.

listed in table 5 (values in brackets). When comparing these numbers with results form the individual optimisations (non-bracketed values in table 5 it becomes clear that the individual design optimisations consistently overestimated each farm's potential. Compared to the designs from the individual optimisations, the differences are marginal in farm 1 and 4 . This can be explained from the fact that these are fairly far apart and not directly in each others wake. The interaction between farm 2 and 3 is stronger however, due to their close proximity and alignment directly in each other's wake. The fact that farm 3 is more affected than farm 2 when taking other farms into account in the optimisation can be ascribed to differences in geometry. The flow through farm 2 is much more constrained due to the presence of the island of Stroma, whereas in the case of farm 3 additional turbines can easily lead to a redirection of the flow northward of the farm which would also redirect it around Stroma island and thus farm 2.

In summary, the influence of multiple farms on one another can be significant, in particular if two farms are in close proximity and share the same tidal stream. This changes both the predicted profit and power production, as well as the optimal tidal farm design. The enhanced computational efficiency of the continuous turbine approach presented in this work facilitates its use in the study of fully coupled multi-farm optimisation.

\section{Farm optimisation with complex constraints}

The practical design of a tidal farm is constrained by various restrictions. For example, turbines may not be able to be placed at locations where the bathymetry has a high slope, as well as in water that is either too shallow or too deep. These sorts of restrictions must be considered when identifying suitable areas for turbine installation. When masking out the site areas which do not adhere to these constraints, one obtains a map where turbines may be placed. 
Figure 10a shows such a map for farm 2 in the Inner Sound, with a maximum steepness condition $\|\nabla h\|<0.015$. The map consists of many disjoint patches of feasible turbine locations. In the discrete farm design approach (Funke et al. 2014), optimising such a site would be extremely challenging since the control space is not convex. The continuous turbine approach however handles this case naturally.

To demonstrate this, we solved the continuous farm optimisation problem on the masked farm area shown in 10a. Otherwise, the problem setup was equivalent to that in section 8 .

The convergence of the optimisation algorithm is shown in figure $10 \mathrm{~b}$ It demonstrates the efficiency of the approach: after 10 iterations, the change in the goal quantity per iteration has dropped below $3 \times 10^{-7}$. Overall, the optimisation was run for 54 shallow-water solves and 52 adjoint shallow-water solves, but clearly could have been stopped earlier.

Finally, figure 9 visualises the flow speed before and after the farm installation. The optimal turbine density is plotted in figures $10 \mathrm{c}$ and $10 \mathrm{~d}$. The optimisation algorithm positioned the areas of high turbine density (red) near the farm boundaries and as barrages across two boundaries, with much of the friction at the east and west sides of the farm and few turbines in the farm interior. The streamlines displayed in these plots show that these barrages are aligned perpendicular to the flow. This has already been observed to be a characteristic of the optimal designs obtained in Funke et al. (2014). Also note that the constraints resulted in a significantly different design with finer structures compared to the non-constrained optimisation (figure 8c). The average power extraction of the optimal farm is $287.9 \mathrm{MW}$. The cost of the farm (measured in power) is $124.6 \mathrm{MW}$. The goal quantity (the profit generating power extraction) is $163.3 \mathrm{MW}$. The estimated number of turbines was 275 , which corresponds to an average power extraction of $1.05 \mathrm{MW}$ per turbine. Comparing these values with the values from the unrestricted optimisation (table 5) shows that the restrictions led to a slight negative impact in the profit and power production of the farm.

\section{Conclusions}

We formulated and solved the problem of finding the most profitable design of tidal stream turbine farms as a mathematical optimisation problem constrained by the shallow water equations. We introduced a continuous approach that describes the farm design using a turbine density function, and demonstrated that this approach is consistent with an earlier approach where turbines are resolved explicitly. The key benefits of this new continuous approach are its computational efficiency, its flexibility and the robustness with respect to incorporating complex farm constraints.

The proposed approach can be used to answer a variety of questions: What is the theoretically maximum extractable power of a tidal site? Taking into account installation costs, what is the maximum overall profit of a farm? To 
achieve this profit, what are the optimal number of turbines, and their positioning within the farm? How do multiple tidal turbine farms impact upon each other?

So far, the proposed methodology has only been applied to the depthaveraged shallow water equations, but it generalises also to fully three-dimensional models. Considering the limitations of a depth-averaged model in predicting tidal streams and in assessing tidal stream energy, future work should apply the same methodology in a three-dimensional setting.

\section{Acknowledgements}

This work was supported by the Research Council of Norway through a Centres of Excellence grant to the Center for Biomedical Computing at Simula Research Laboratory (project number 179578) and the UK's Engineering and Physical Science Research Council (projects: EP/J010065/1 and EP/M011054/1). Computations were performed on the Abel supercomputing cluster at the University of Oslo via NOTUR project NN9279K and NN9316K. The authors would like to thank M. Nordaas for valuable discussions. We would like to thank MeyGen Limited for supplying high resolution bathymetry data and ADCP data in the Inner Sound area.

\section{References}

Adcock, T.A.A., Borthwick, A.G.L., Houlsby, G.T., 2011. The open boundary problem in tidal basin modelling with energy extraction, in: Proceedings of the 9th European Wave and Tidal Energy Conference. Southampton, UK.

Ahmadian, R., Falconer, R.A., 2012. Assessment of array shape of tidal stream turbines on hydro-environmental impacts and power output. Renewable Energy 44, 318 - 327. doi $10.1016 /$ j.renene.2012.01.106.

Andritz Hydro Hammerfest, 2012. Renewable energy from tidal currents. URL: http://www. andritz.com/hy-hammerfest.pdf.

Barnett, G.L., Funke, S.W., Piggott, M.D., 2015. Hybrid global-local optimisation algorithms for the layout design of tidal turbine arrays. Renewable Energy Submitted.

British Oceanographic Data Centre (BODC), 2010. GEBCO_08 bathymetry grid, version 20100927. http://www.gebco.net.

Byrd, R.H., Lu, P., Nocedal, J., Zhu, C., 1995. A limited memory algorithm for bound constrained optimization. SIAM Journal on Scientific and Statistical Computing 16, 1190-1208. doi:10.1137/0916069.

Culley, D.M., Funke, S.W., Kramer, S.C., Piggott, M.D., 2014. A hierarchy of approaches for the optimal design of tidal turbine arrays. ICOE 2014 (5th International Conference on Ocean Energy) . 
Culley, D.M., Funke, S.W., Kramer, S.C., Piggott, M.D., 2016. Integration of cost modelling within the micro-siting design optimisation of tidal turbine arrays. Renewable Energy 85, 215 - 227. doi $10.1016 / j$.renene.2015.06.13.

Divett, T., Vennell, R., Stevens, C., 2013. Optimization of multiple turbine arrays in a channel with tidally reversing flow by numerical modelling with adaptive mesh. Philosophical Transactions of the Royal Society of London A: Mathematical, Physical and Engineering Sciences 371. doi:10.1098/rsta. 2012.0251

Draper, S., Adcock, T.A.A., Borthwick, A.G.L., Houlsby, G.T., 2013. An electrical analogy for the Pentland Firth tidal stream power resource. Proceedings of the Royal Society of London A: Mathematical, Physical and Engineering Sciences 470. doi $10.1098 /$ rspa.2013.0207.

Draper, S., Adcock, T.A.A., Borthwick, A.G.L., Houlsby, G.T., 2014. Estimate of the tidal stream power resource of the Pentland Firth. Renewable Energy 63, 650-657. doi $10.1016 /$ j.renene.2013.10.015.

Easton, M.C., Woolf, D.K., Bowyer, P.A., 2012. The dynamics of an energetic tidal channel, the Pentland Firth, Scotland. Continental Shelf Research 48, 50-60. doi $10.1016 /$ j.csr.2012.08.009.

Edina Digimap service, 2014. Hydrospatial one, gridded bathymetry. http: //edina.ac.uk/digimap/, downloaded 2014.

Egbert, G., Erofeeva, L., 2010. OSU Tidal Prediction Software (OTPS). http: //volkov.oce.orst.edu/tides/otps.html

Farrell, P.E., Ham, D.A., Funke, S.W., Rognes, M.E., 2013. Automated derivation of the adjoint of high-level transient finite element programs. SIAM Journal on Scientific Computing doi:10.1137/120873558

Funke, S.W., 2013. The automation of PDE-constrained optimisation and its applications. Ph.D. thesis. Imperial College London. London, UK.

Funke, S.W., Farrell, P.E., Piggott, M.D., 2014. Tidal turbine array optimisation using the adjoint approach. Renewable Energy 63, 658 - 673. doi10.1016/ j.renene.2013.09.031.

Garrett, C., Cummins, P., 2005. The power potential of tidal currents in channels. Proceedings of the Royal Society A: Mathematical, Physical and Engineering Science 461, 2563-2572. doi 10.1098/rspa.2005.1494.

Garrett, C., Cummins, P., 2008. Limits to tidal current power. Renewable Energy 33, 2485-2490. doi $10.1016 /$ j.renene.2008.02.009.

Kramer, S.C., Piggott, M.D., 2015. A correction to the enhanced bottom drag parameterisation of tidal turbines. Renewable Energy, Submitted. 
Lee, S.H., Lee, S.H., Jang, K., Lee, J., Hur, N., 2010. A numerical study for the optimal arrangement of ocean current turbine generators in the ocean current power parks. Current Applied Physics 10, S137 - S141. doi 10.1016/j.cap. 2009.11.018.

Logg, A., Mardal, K.A., Wells, G.N., 2012. Automated Solution of Differential Equations by the Finite Element Method. Springer. doi doi: 10.1007/978-3-642-23099-8.

Martin-Short, R., Hill, J., Kramer, S.C., Avdis, A., Allison, P.A., Piggott, M.D., 2015. Tidal resource extraction in the Pentland Firth, UK: Potential impacts on flow regime and sediment transport in the Inner Sound of Stroma. Renewable Energy 76, 596 - 607. doi:10.1016/j.renene.2014.11.079

Nishino, T., Willden, R.H.J., 2012. The efficiency of an array of tidal turbines partially blocking a wide channel. Journal of Fluid Mechanics 708, 596-606.

Pawlowicz, R., Beardsley, B., Lentz, S., 2002. Classical tidal harmonic analysis including error estimates in MATLAB using T_TIDE. Computers \& Geosciences 28, 929-937. doi 10.1016/S0098-3004(02)00013-4.

Polagye, B.L., Malte, P.C., 2011. Far-field dynamics of tidal energy extraction in channel networks. Renewable Energy 36, 222-234. doi http://dx.doi. org/10.1016/j.renene.2010.06.025.

Roc, T., Funke, S.W., Thyng, K.M., 2015. Standard methodology for tidal array project optimisation: An idealized study of the Minas passage, in: Proceedings of the 11th European Wave and Tidal Energy Conference - EWTEC 2015.

Scottish Goverment, 2009. Pentland Firth bathymetry. http: //www.scotland.gov.uk/Topics/marine/science/MSInteractive/ datatype/Bathymetry/data/PentlandFirthBathymetry.

Sutherland, G., Foreman, M., Garrett, C., 2007. Tidal current energy assessment for Johnstone Strait, Vancouver Island, in: Proceedings of the Institution of Mechanical Engineers A 221, pp. 147-157.

The Crown Estate, 2013. Pentland Firth and Orkney waters strategic area review. http://www.thecrownestate.co.uk/media/432929/ pfow-strategic-area-review-project-2012.pdf.

UKHO, 2009. Admirality tide table: United Kingdom and Ireland, including European Channel ports. 1.

Vennell, R., 2010. Tuning turbines in a tidal channel. Journal of Fluid Mechanics 663, 253-267. doi $10.1017 /$ S0022112010003502.

Vennell, R., 2011. Estimating the power potential of tidal currents and the impact of power extraction on flow speeds. Renewable Energy 36, 3558-3565. doi $10.1016 /$ j.renene.2011.05.011. 
Vennell, R., Funke, S.W., Draper, S., Stevens, C., Divett, T., 2015. Designing large arrays of tidal turbines: A synthesis and review. Renewable and Sustainable Energy Reviews 41, $454-472$. doi:10.1016/j.rser.2014.08.022.

Vogel, C.R., 2014. Theoretical limits to tidal stream energy extraction. Ph.D. thesis. University of Oxford.

Whelan, J.I., Graham, J.M.R., Peiró, J., 2009. A free-surface and blockage correction for tidal turbines. Journal of Fluid Mechanics 624, 281-291. doi:10 . 1017/S0022112009005916.

Zhu, C., Byrd, R.H., Lu, P., Nocedal, J., 1997. Algorithm 778: L-BFGS-B: Fortran subroutines for large-scale bound-constrained optimization. ACM Transactions on Mathematical Software 23, 550-560. doi $10.1145 / 279232$. 279236. 


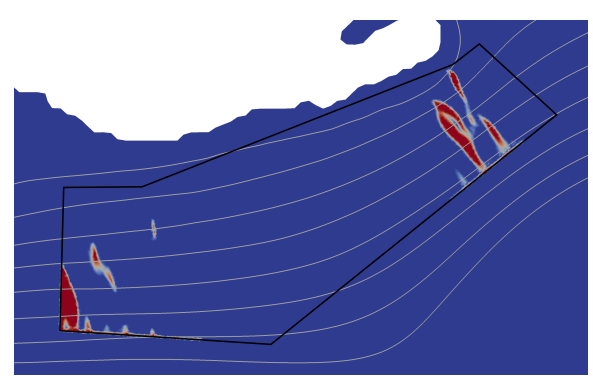

(a) Farm 1 (228 turbines)

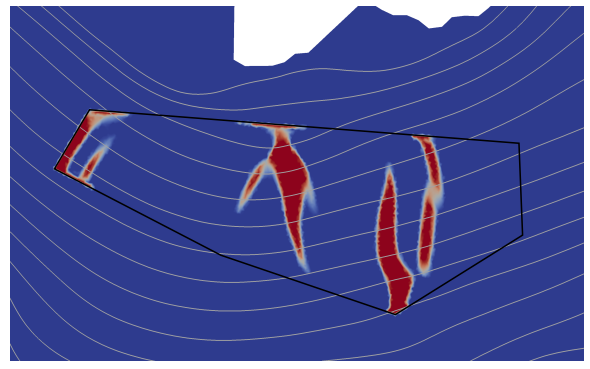

(c) Farm 2 (266 turbines)

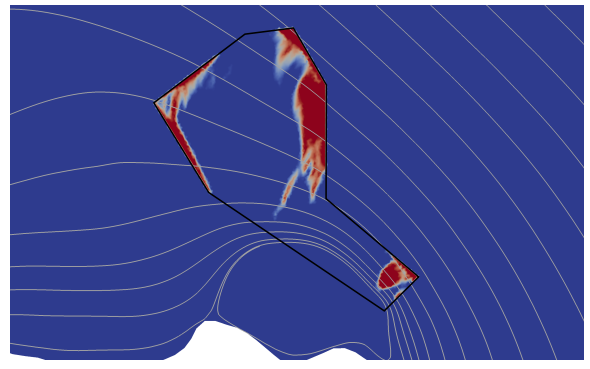

(e) Farm 3 (249 turbines)

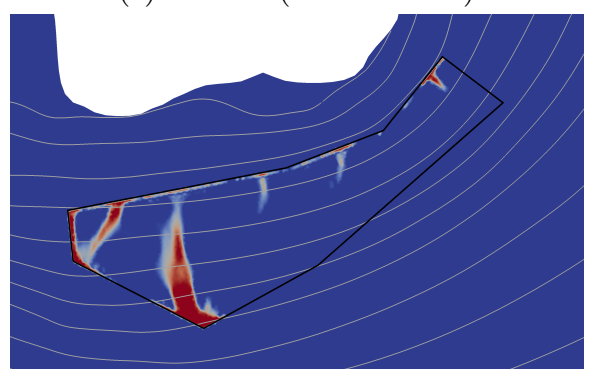

(g) Farm 4 (156 turbines)

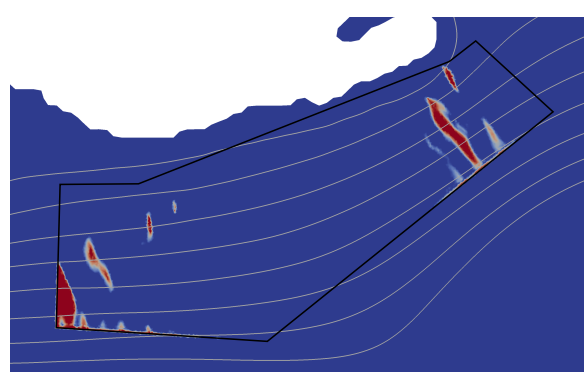

(b) Farm 1 (224 turbines)

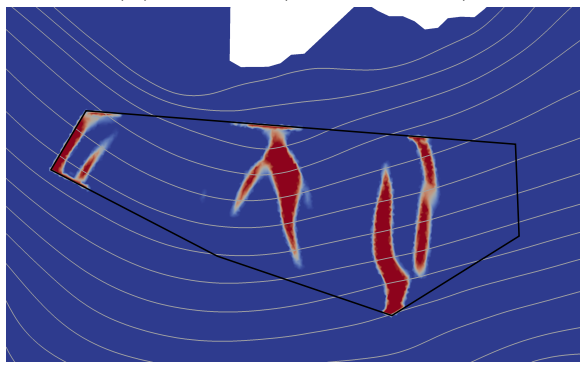

(d) Farm 2 (251 turbines)

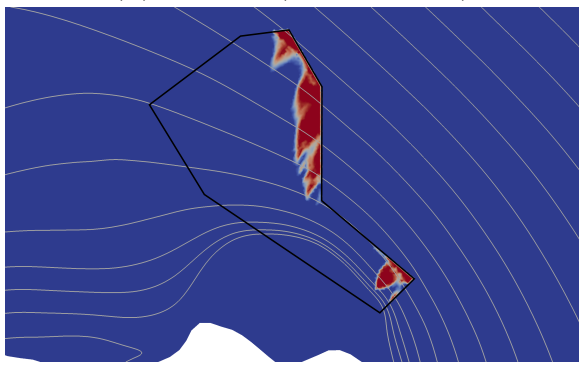

(f) Farm 3 (162 turbines)

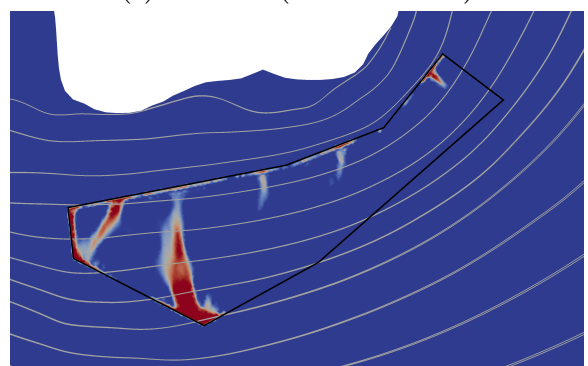

(h) Farm 4 (153 turbines)

Figure 8: The optimal turbine densities for the four farms in the Pentland Firth. The designs on the left were obtained by individually optimising each farm, not taking into account the existence of the other farms, while the designs on the right were obtained from a multi-farm optimisation to maximise the overall profit of all farms. 

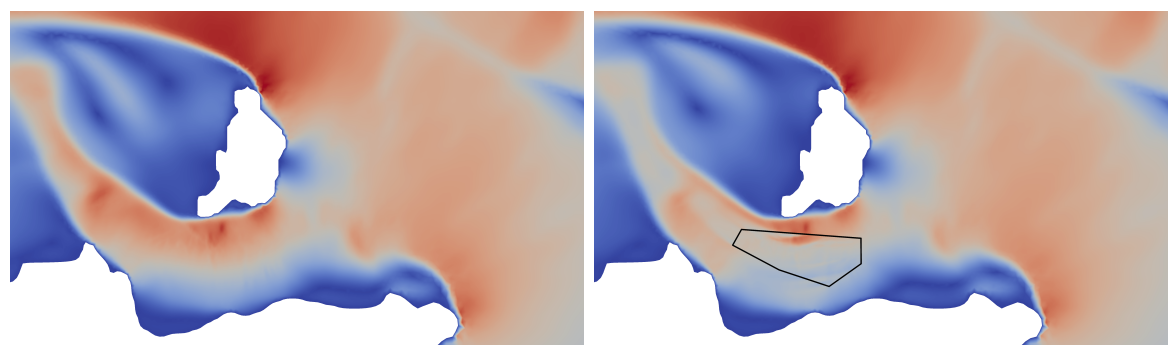

(a) Flow speed without farm during ebb

(b) Flow speed with farm during ebb
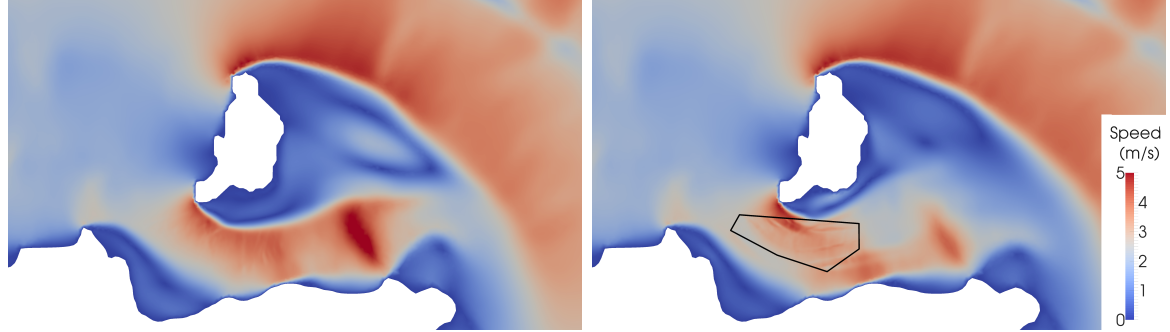

(c) Flow speed without farm during flood

(d) Flow speed with farm during flood

Figure 9: The results of the farm optimisation with complex constraints

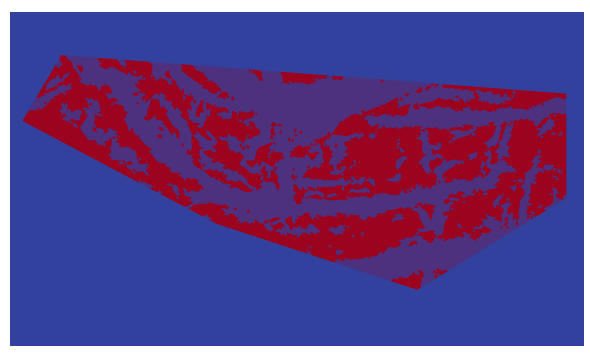

(a) Original (shaded red) and masked (red) turbine farm area

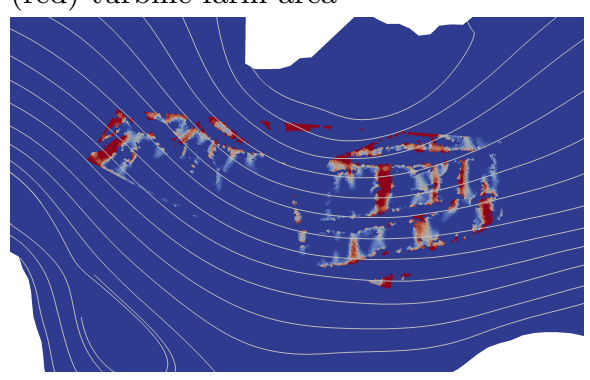

(c) Optimal turbine density with stream (d) Optimal turbine density with stream lines during flood

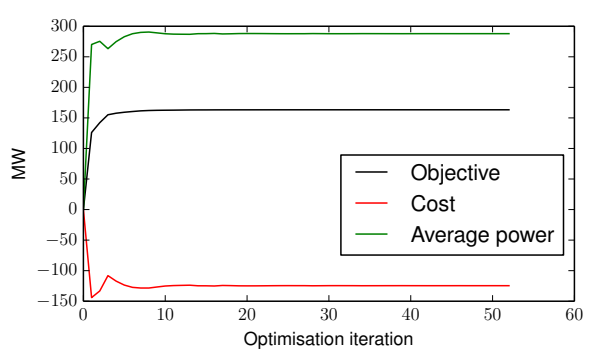

(b) Optimisation iterations

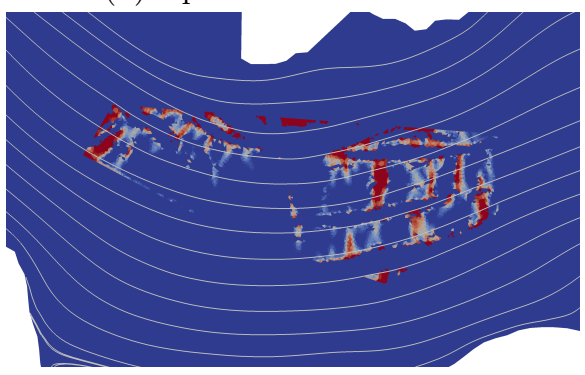
lines during ebb

Figure 10: The results of the farm optimisation with complex constraints 